\title{
Tris-(2,3-dibromopropyl) isocyanurate induces depression-like behaviors and neurotoxicity by oxidative damage and cell apoptosis in vitro and in vivo
}

\author{
Zhaoju Dong', Zhengping Hu${ }^{3}$, Haibo Zhu', ${ }^{1,2}$ Ning Li', Huijuan Zhao', Wei Mi', \\ Wanglin Jiang ${ }^{4}$, Xihou Hu${ }^{1}$ and Liang $\mathrm{Ye}^{1,2}$ \\ ${ }^{1}$ School of Public Health and Management, Binzhou Medical University, Yantai, Shandong, 264003, China \\ 2Institute of Toxicology, Binzhou Medical University, Yantai, Shandong, 264003, China \\ ${ }_{3}^{3}$ Medicine \& Pharmacy Research Center, Binzhou Medical University, Yantai, Shandong, 264003, China \\ ${ }^{4}$ School of Pharmacy, Binzhou Medical University, Yantai, Shandong, China
}

(Received May 29, 2015; Accepted August 17, 2015)

\begin{abstract}
Tris-(2,3-dibromopropyl) isocyanurate (TDBP-TAZTO), an emerging brominated flame retardant, possesses the characteristics of candidate persistent organic pollutants and has displayed toxicity to fish and rodents. TDBP-TAZTO can pass through the blood-brain barrier and accumulate in the brain. TDBP-TAZTO might also induce neuronal cell toxicity. However, the neurotoxicity and mechanisms of TDBP-TAZTO have not yet been studied. We hypothesize that TDBP-TAZTO could induce neurotoxicity in mouse hippocampal neurons and SH-SY5Y cells. The mice were exposed to TDBP-TAZTO of 5 and $50 \mathrm{mg} / \mathrm{kg}$ by gavage, daily for 30 days. TDBP-TAZTO resulted in depression-like behaviors, which may be related with TDBP-TAZTO-induced upregulation of oxidative stress markers and overexpression of pro-apoptotic proteins in hippocampus. Furthermore, TDBP-TAZTO treatment for $48 \mathrm{hr}(12.5$, 25 and $50 \mu \mathrm{M}$ ) damaged SH-SY5Y cells, and led to cell apoptosis and oxidative stress in concentrationdependent manner. Our findings suggested that cell apoptosis and oxidative stress are important mechanisms in neurotoxicity induced by TDBP-TAZTO.
\end{abstract}

Key words: Tris-(2,3-dibromopropyl) isocyanurate (TDBP-TAZTO), Depression, Hippocampus, Oxidative stress, Apoptosis

\section{INTRODUCTION}

Brominated flame retardants (BFRs) have been widely used to reduce fire-related injury and property damage in plastics, textiles, synthetic fibers and other materials (Li et al., 2015; Feng et al., 2010). However, some BFRs, such as tetrabromobisphenol-A and polybrominated diphenylethers, can accumulate in the human body and affect human health. The accumulating data shown that some BFRs can induce neurotoxicity, immunotoxicity and endocrine toxicity in rodents (Nakajima et al., 2009; Wang et al., 2013; Dingemans et al., 2011). The pentaand octa-BDE mixtures has been listed in the Stockholm Convention on Persistent Organic Pollutants (POPs) (Zhu et al., 2012). Currently, the demand for alternative BFRs, such as tris-(2,3-dibromopropyl) isocyanurate (TDBPTAZTO), has steadily increased (Feng et al., 2010).
The first reported use of TDBP-TAZTO appeared as a flame retardant in styrene polymers in 1959, and it was used in Europe and Japan. The annual production volume of TDBP-TAZTO in China in the 1990s was more than 500 metric tons (Li et al., 2011). Following the cessation of commercial production of some BFRs worldwide, the increasing production and use of TDBP-TAZTO may be expected in Europe, the US and Japan, among other places (Zhu et al., 2012).

TDBP-TAZTO, which has recently been identified in soils, sediments, and earthworms, is considered persistent and can bioaccumulate in some organisms (Ruan et al., 2009; Zhu et al., 2012). Especially, the intestine and brain of the observed carp contained high levels of TDBP-TAZTO, which indicated that this substance can pass through the blood-brain barrier (Ruan et al., 2009). The studies on zebrafish embryos showed that TDBP-TAZTO can cause

Correspondence: Liang Ye (E-mail: project0088@hotmail.com) 
the potential reproductive and endocrine disrupting toxic effects, damaging mitochondria and causing defects in gas bladder inflation (Zhang et al., 2011; Li et al., 2011). Another study indicated that TDBP-TAZTO can induce significant toxicity in mice organs, especially in the liver and lung possibly (Li et al., 2015). In addition, TDBPTAZTO might also induce neuronal cell toxicity (Qu et al., 2011). Our study found that TDBP-TAZTO of 5 and $50 \mathrm{mg} / \mathrm{kg}$ by gavage, daily for 6 months, resulted in cognitive impairment and depression-like behaviors in the male adult rats. And TDBP-TAZTO induces significant hippocampal neurotoxicity, characterized by upregulating inflammatory and oxidative stress markers, and promoting cell apoptosis in the hippocampus (data submitted to another journal).

In order to further elucidate the mechanisms of TDBPTAZTO-induced depression-like behaviors, we investigate if TDBP-TAZTO induces neurotoxicity by promoting oxidative stress and apoptosis in vitro and in vivo in the present study.

\section{MATERIALS AND METHODS}

\section{Materials and drugs}

TDBP-TAZTO (purity $>97 \%$, molecular structure shown in Fig. 1), propidium iodide (PI, Cat. no. P4170), dimethyl sulfoxide (DMSO, Cat. no. D8418), and 3-(4,5Dimethylthiazol-2-yl)-2,5-diphenyltetrazolium bromide (MTT) were provided by Sigma-Aldrich (St. Louis, MO, USA). The primary rabbit antibodies to Bcl-2 (Cat. no. 2876) and Bax (Cat. no. 2772S) were provided by Cell Signaling Technology (Beverly, MA, USA). Goat anti-rabbit IgG-HRP secondary antibodies (Cat. no. A0208 and A3216) were provided by Beyotime Biotechnology, Qindao, China. The assay kits of superoxide dismutase (SOD, Cat. no. S0060), maldondialdehyde (MDA, Cat. no. S0131) and glutathione (GSH,

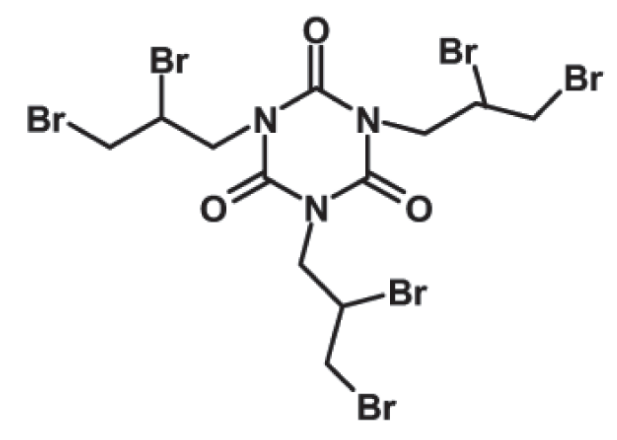

Fig. 1. Molecular structure of TDBP-TAZTO.
Cat. no. A006) were provided by the Nanjing Jiancheng Bioengineering Institute, Nanjing, China. Dulbecco's modified Eagle's medium (DMEM), fetal bovine serum (FBS) were provided by Hyclone Company (Logan, UT, USA). Proteinase K was purchased by Merck Corp., Darmstadt, Germany.

\section{Animals}

Male Balb/c mice (19-22 g) were obtained from Beijing Weitong Lihua Experimental Animal Centre and were acclimatized for at least 5 days before use in the experiments. All the animals were housed under constant environmental conditions $\left(22 \pm 2^{\circ} \mathrm{C}, 40-70 \%\right.$ relative humidity) with a $12: 12 \mathrm{hr}$ light/dark cycle (lights were on at 06:00 and off at 18:00) and were given free access to uniform food and sterile water. This experiment was approved by the Ethics Committee of Binzhou Medical University (No. 013 in 2014 for Animal Ethics Approval). The local legislation regarding the ethics of animal experimentation and the guidelines for the care and use of laboratory animals were followed in all animal procedures. All surgery was performed under anesthesia, and all efforts were made to minimize suffering.

\section{TDBP-TAZTO exposure to mice}

The male mice were randomly assigned to three groups (10/group). TDBP-TAZTO was dissolved in corn oil for the experiments. Mice were exposed to TDBP-TAZTO of $5 \mathrm{mg} / \mathrm{kg}$ or $50 \mathrm{mg} / \mathrm{kg}$, or corn oil by gavage from 8 a.m. to 9 a.m. for 30 days. The open field test, the tail suspension test and the forced swimming test were conducted $60 \mathrm{~min}$ after TDBP-TAZTO treatment on the $28^{\text {th }}, 2^{\text {th }}$ and $30^{\text {th }}$ day, respectively.

\section{The open field test in mice}

To evaluate the possible effects of TDBP-TAZTO on locomotor activity, mice were individually placed in the plastic box $(50 \times 50 \times 50 \mathrm{~cm})$. The moving distance in 20 -min test was automatically recorded by a video camera and analyzed by using Topscan system (Clever Sys., Inc., Reston, VA, USA). The boxes were cleaned between the animals.

\section{Forced swimming test in mice}

Forced swimming test (FST) was performed according to the previous method (Ye et al., 2012). Briefly, the animal was individually forced to swim in a glass cylinder ( $25 \mathrm{~cm}$ high, $12 \mathrm{~cm}$ in diameter) filled with water $\left(22 \pm 2{ }^{\circ} \mathrm{C}\right)$ to a depth of $15 \mathrm{~cm}$. FST were videotaped from the side of the cylinder. The total duration of immobility was measured during $6 \mathrm{~min}$. Immobility was defined 
TDBP-TAZTO induces depression-like behaviors and neurotoxicity

as floating in the water and treading water just enough to keep the nose above water (Galdino et al., 2009). The data were analyzed by using DepressionScan system (Clever Sys., Inc., USA).

\section{Tail suspension test in mice}

Tail suspension test (TST) was performed according to the previous described method (Ye et al., 2012) with minor modification. Briefly, the mice were individually suspended $50 \mathrm{~cm}$ above the surface of table by fixing the tail using adhesive tape. The tests were videotaped and the duration of immobility was recorded during the last 4 min of the total 6-min test. Immobility was defined as when they hung passively and were completely motionless (Galdino et al., 2009). The data were analyzed by using DepressionScan system.

\section{Dissection and homogenization}

All the mice were decapitated between 9:00 a.m. and 11:00 a.m. The removed brains were placed on dry ice for hippocampus dissection and 10\% (w/v) hippocampus homogenates were prepared in $0.1 \mathrm{M}$ phosphate buffer ( $\mathrm{pH}$ 7.4) with or without protease inhibitor cocktails. The homogenates were centrifuged at $10,000 \mathrm{~g}$ for $10 \mathrm{~min}$ and the supernatants were collected for the oxidative stress markers determination, or western blotting analysis.

\section{SH-SY5Y cell culture and TDBP-TAZTO exposure}

SH-SY5Y cells (neuroblastoma cell line) were maintained in DMEM medium supplemented with 10\% FBS in a humidified incubator with $5 \% \mathrm{CO}_{2}$ at $37^{\circ} \mathrm{C}$. The cells were seeded in 96-cell plates for $24 \mathrm{hr}$, and incubated with TDBP-TAZTO solution for another $48 \mathrm{hr}$. TDBP-TAZTO was dissolved in DMSO. The final concentrations in plates were $6.25,12.5,25,50$ and $100 \mu \mathrm{M}$, respectively, and the final DMSO concentration was less than $0.1 \%$.

\section{SH-SY5Y cells viability assay}

Cell viability was determined by MTT assay. After TDBP-TAZTO exposure for $48 \mathrm{hr}, 20 \mu \mathrm{L}$ MTT $(5 \mathrm{mg} / \mathrm{mL})$ was added to each well and incubated for $4 \mathrm{hr}$ and $150 \mu \mathrm{L}$ DMSO was added into each well after the medium was removed. The optical density (OD) was determined by using SpectraMax M5 multi-detection reader (Molecular Devices, Inc., Sunnyvale, CA, USA) at $490 \mathrm{~nm}$. Cell viability was presented as percentage of OD values in comparison with the control.

\section{Analysis of DNA fragmentation of SH-SY5Y cells by agarose gel electrophoresis}

SH-SY5Y cells were plated at $3 \times 10^{5}$ per well in 6-well plates. After $24 \mathrm{hr}$, cells were treated with TDBPTAZTO $(12.5,25$ and $50 \mu \mathrm{M})$ for $48 \mathrm{hr}$. DNA fragmentation was determined by extraction of DNA followed by agarose gel electrophoresis. In brief, cells were pelleted, washed twice with PBS, resuspended in the extraction buffer (10 mM Tris, 0.1 M EDTA, $20 \mu \mathrm{g} / \mathrm{mL}$ trypsin, $5 \mathrm{mg} / \mathrm{mL} \mathrm{SDS} ; \mathrm{pH} \mathrm{8.0)}$ and incubated for $1 \mathrm{hr}$ at $37^{\circ} \mathrm{C}$. The homogenates were then incubated with $20 \mathrm{mg} /$ $\mathrm{mL}$ proteinase $\mathrm{K}$ (Merck) for $3 \mathrm{hr}$ at $50^{\circ} \mathrm{C}$. After phenol extraction, DNA was precipitated, dissolved in the TE buffer and electrophoresed in $1.5 \%$ agarose gel. DNA ladder formation was visualized by ethidium bromide staining under UV light.

\section{SH-SY5Y cell extract preparation}

SH-SY5Y cells were plated at $3 \times 10^{5}$ per well in 6-well plates. After $24 \mathrm{hr}$, cells were treated with TDBPTAZTO $(12.5,25$ and $50 \mu \mathrm{M})$ for $48 \mathrm{hr}$. Cell extracts were prepared with or without protease inhibitor cocktails or the oxidative stress markers determination, or western blotting analysis.

\section{Apoptosis-related proteins in mouse hippocampus and SH-SY5Y cells}

The protein content of the hippocampal homogenates or cell extracts were measured by BCA method. The same amounts of proteins (20-30 $\mu \mathrm{g}$ per lane) were separated by SDS polyacrylamide gel electrophoresis, and transferred onto a nitrocellulose membrane. These membranes were incubated with $1 \%$ bovine serum albumin in Tween-Tris-buffered saline and reacted overnight at $4{ }^{\circ} \mathrm{C}$ with the rabbit antibody (1:1000) against Bcl-2 and Bax. After repeated washings, the immunoreactive bands were reacted with horseradish peroxidase-conjugated anti-rabbit antibody, then visualized by FluorChem ${ }^{\mathrm{TM}} \mathrm{Q}$ chemiluminescent System (ProteinSimple Corp., Toronto, ON, Canada). The O.D. value of reactive bands visible was determined densitometrically. $\beta$-actin was used as an internal control.

\section{Oxidative stress markers in mouse hippocampus and SH-SY5Y cells}

The following oxidative stress markers in the mouse hippocampus and SH-SY5Y cells, such as SOD activity, GSH and MDA content were determined by the biochemical assay kits according to manufacturer instructions.

\section{Statistical analyses}

The statistical analyses were performed using one-way ANOVA, followed by LSD post hoc test in SPSS software (V 16.0). $P<0.05$ was considered statistically significant. 


\section{RESULTS}

\section{Effects of TDBP-TAZTO on the behaviors in the FST, TST and locomotor activity}

In order to determine if TDBP-TAZTO can induce depression-related behavior, both FST and TST were conducted after TDBP-TAZTO treatment for 30 days. The results showed that TDBP-TAZTO at $5 \mathrm{mg} / \mathrm{kg}$ and $50 \mathrm{mg} / \mathrm{kg}$ dose-dependently increased the duration of immobility in the mice FST, as compared with control group (each $p<0.05$ ) (Fig. 2A). Likewise, as shown in Fig. 2B, TDBP-TAZTO at $5 \mathrm{mg} / \mathrm{kg}$ and $50 \mathrm{mg} / \mathrm{kg}$ had significantly increased the duration of immobility compared with control mice in the TST. However, as seen in Fig. 2C, TDBP-TAZTO of 5 and $50 \mathrm{mg} / \mathrm{kg}$ did not produce significant effects on the moving distance compared with control group (each $p>0.05$ ).

\section{Effects of TDBP-TAZTO on apoptosis-related proteins in mouse hippocampus}

To evaluate the possible effects of TDBP-TAZTO on nerve cell apoptosis in the mouse hippocampus after 30-day exposure, the expression of the anti-apoptotic protein $\mathrm{Bcl}-2$ and the pro-apoptotic protein Bax were measured by the Western blotting method. As shown in Fig. 3, there was a main effect of TDBP-TAZTO in levels of Bax and bcl-2 normalized to $\beta$-actin (each $p<0.05$ ). TDBP-TAZ$\mathrm{TO}$ of $50 \mathrm{mg} / \mathrm{kg}$ significantly increased Bax level, while decreased bcl-2 levels compared to control group (each $p<0.05)$.

\section{Effects of TDBP-TAZTO on oxidative stress markers in mouse hippocampus}

To investigate the possible the effects of TDBP-TAZ-
TO on oxidative stress in the mouse hippocampus, several oxidative stress-related markers were measured by the biochemical assay kits. As shown in Table 1, compared with control group, TDBP-TAZTO significantly increased MDA level (each $p<0.05$ ), and decreased GSH level (each $p<0.05$ ) and SOD activity (each $p<0.05$ ) in a dose-dependent manner in the mouse hippocampus.

\section{Effects of TDBP-TAZTO on SH-SY5Y cells survival}

To further confirm the damage effects and the mechanisms of TDBP-TAZTO on neuronal cells, the effects of TDBP-TAZTO on SH-SY5Y cells survival, apoptosis, and oxidative stress were assessed. First, MTT assay was used to investigate the effects of TDBP-TAZTO on the viability of SH-SY5Y cells. As shown in Fig. 4, at concentration from 12.5 to $100 \mu \mathrm{M}$, TDBP-TAZTO resulted in a concentration-dependent reduction in cell viability.

\section{DNA fragmentation of SH-SY5Y cells induced by TDBP-TAZTO}

In order to investigate the underlying mechanism responsible for the growth-inhibitory effect of TDBPTAZTO, genomic DNA fragmentation was analyzed by agarose gel electrophoresis. As seen in Fig. 5, treatment with TDBP-TAZTO of $12.5,25$ and $50 \mu \mathrm{M}$ for $48 \mathrm{hr}$ produced evident DNA laddering, a typical subcellular symptom of apoptosis.

\section{Effects of TDBP-TAZTO on apoptosis-related proteins in SH-SY5Y cells}

To further provide the mechanisms of apoptosis, the expression of bcl-2 and Bax were determined by western blotting analysis after the SH-SY5Y cells were treat-
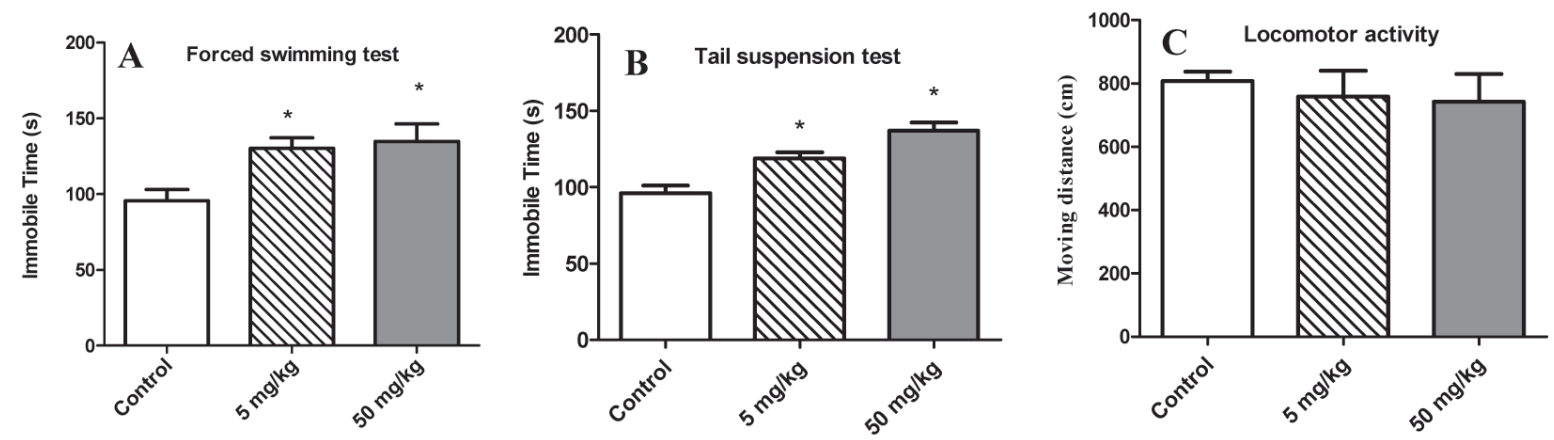

Fig. 2. The effects of TDBP-TAZTO (5 and $50 \mathrm{mg} / \mathrm{kg}$ ) on the durations of immobility in FST (A) and TST (B), and the locomotor activity $(\mathrm{C})$ in mice. In FST, the animal was individually forced to swim in a glass cylinder and the total duration of immobility was measured during $6 \mathrm{~min}$. In TST, the mice were individually suspended $50 \mathrm{~cm}$ above the surface of table by fixing the tail using adhesive tape, the duration of immobility was recorded during the last 4 min of the total 6-min test. In the open field test, the mice were individually placed in the plastic box and the moving distance in 20-min test was automatically recorded. The results are presented as mean \pm S.E. $\left(n=10 /\right.$ group). ${ }^{*} p<0.05$, vs. control group. 
TDBP-TAZTO induces depression-like behaviors and neurotoxicity

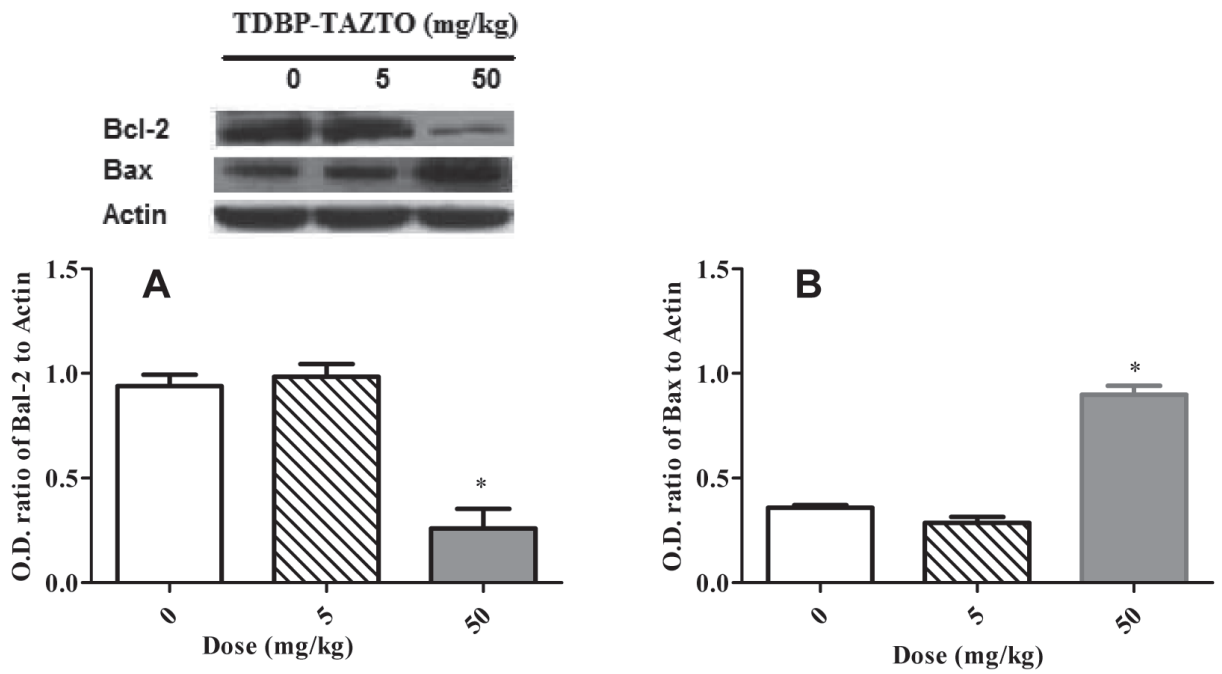

Fig. 3. The effects of TDBP-TAZTO on apoptosis-related proteins in the mouse hippocampus $(\mathrm{n}=5)$. The hippocampal proteins (20-30 $\mu \mathrm{g}$ per lane) were separated by SDS polyacrylamide gel electrophoresis, and transferred onto a nitrocellulose membrane. These membranes were reacted overnight at $4{ }^{\circ} \mathrm{C}$ with the rabbit antibody $(1: 1000)$ against Bcl-2 and Bax. After repeated washings, the immunoreactive bands were reacted with horseradish peroxidase-conjugated anti-rabbit antibody. The O.D. value of reactive bands visible was determined densitometrically. $\beta$-actin was used as internal control. The results are presented as mean \pm S.E. ${ }^{*} p<0.05$, vs. control group.

Table 1. The effects of TBC on oxidative and anti-oxidative enzymes in the mouse hippocampus $(\mathrm{n}=5)$.

\begin{tabular}{lccc}
\hline Group & MDA (nmol/mg pro) & GSH (nmol/mg pro) & SOD (U/mg pro) \\
\hline Control & $0.15 \pm 0.11$ & $65.21 \pm 2.32$ & $1.58 \pm 0.15$ \\
TBC $5 \mathrm{mg} / \mathrm{kg}$ & $0.43 \pm 0.09^{*}$ & $53.15 \pm 2.93^{*}$ & $1.21 \pm 0.15^{*}$ \\
TBC $50 \mathrm{mg} / \mathrm{kg}$ & $0.55 \pm 0.16^{*}$ & $37.67 \pm 1.87^{*}$ & $0.72 \pm 0.22^{*}$ \\
\hline
\end{tabular}

Note: The results are presented as mean \pm S.E. ${ }^{*} p<0.05$, vs. control group.

ed with TDBP-TAZTO for $48 \mathrm{hr}$. The results shown that TDBP-TAZTO dose-dependently increased Bax level, while decreased bcl-2 levels compared to control group (each $p<0.05)$ (Fig. 6).

\section{Effects of oxidative stress markers in SH-SY5Y cells}

In order to clear the effects of TDBP-TAZTO on oxidative stress markers, MDA and GSH content and SOD activity were investigated in SH-SY5Y cells after $48 \mathrm{hr}$ of TDBP-TAZTO treatment. As shown in Table 2, TDBP-TAZTO significantly increased MDA level (each $p<0.05$ ), and decreased GSH level (each $p<0.05$ ) and SOD activity (each $p<0.05$ ) in a dose-dependent manner in SH-SY5Y cells, as compared with control group.

\section{DISCUSSION}

BFRs have been widely applied to prevent or minimize fire hazards. The recent studies indicated that some BFRs can induce neurotoxicity, including some POPs (Dingemans et al., 2011). The adverse effects of BFRs on spontaneous behavior and Morris water maze relearning abilities were widely studied mainly during early neurodevelopment rather than during the adulthood years in rodents. However, few studies showed that BFRs can provoke depression-like behaviors in rodents (Dingemans et al., 2011; Kajta and Wójtowicz, 2013). Our previous study found that TDBP-TAZTO of 5 and $50 \mathrm{mg} / \mathrm{kg}$ resulted in cognitive impairment and depression-like behaviors in the male adult rats by TDBP-TAZTO-induced significant hippocampal neurotoxicity (data submitted to anoth- 


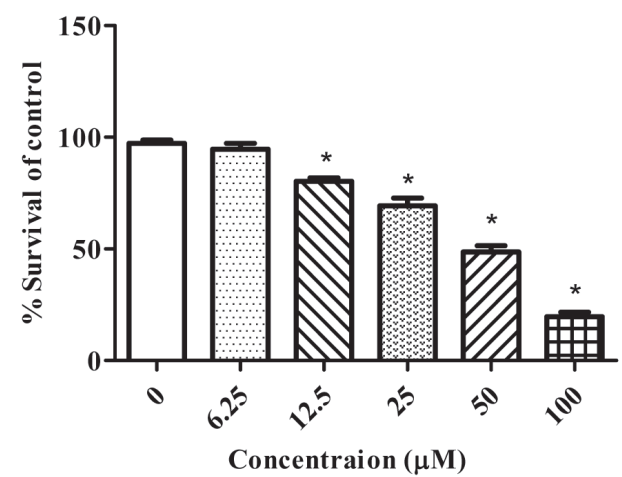

Fig. 4. The effects of TDBP-TAZTO on SH-SY5Y cells survival. Cell were incubated with TDBP-TAZTO of different concentration for $48 \mathrm{hr}$ followed by MTT assay. The OD was determined at $490 \mathrm{~nm}$ and cell survival rate was presented compared with control group. The Data are expressed as mean \pm S.E. of three separate experiments. ${ }^{*} p<0.05$, vs. control group.

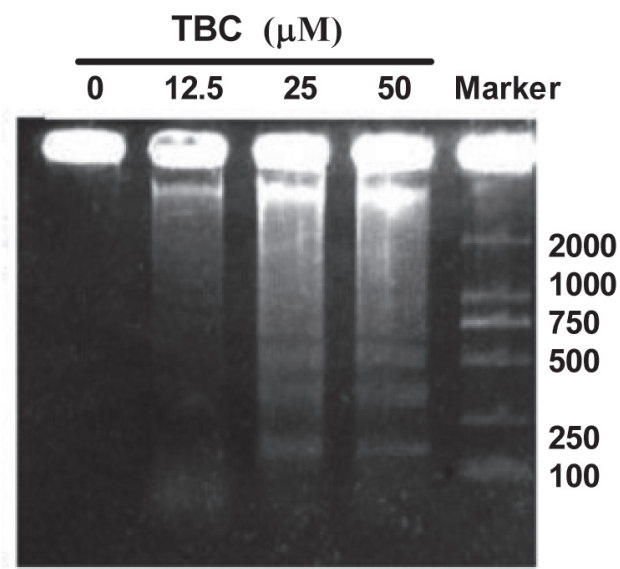

Fig. 5. DNA fragmentation in SH-SY5Y cells was induced by TDBP-TAZTO. After SH-SY5Y cells were treated with the indicated concentrations of TDBP-TAZTO for $48 \mathrm{hr}$. DNA was extracted in the extraction buffer for $1 \mathrm{hr}$ at $37^{\circ} \mathrm{C}$. The homogenates were then incubated with $20 \mathrm{mg} / \mathrm{mL}$ proteinase $\mathrm{K}$ for $3 \mathrm{hr}$ at $50^{\circ} \mathrm{C}$. After phenol extraction, DNA was precipitated, dissolved in the TE buffer and electrophoresed in $1.5 \%$ agarose gel. DNA ladder formation was visualized by ethidium bromide staining under UV light.
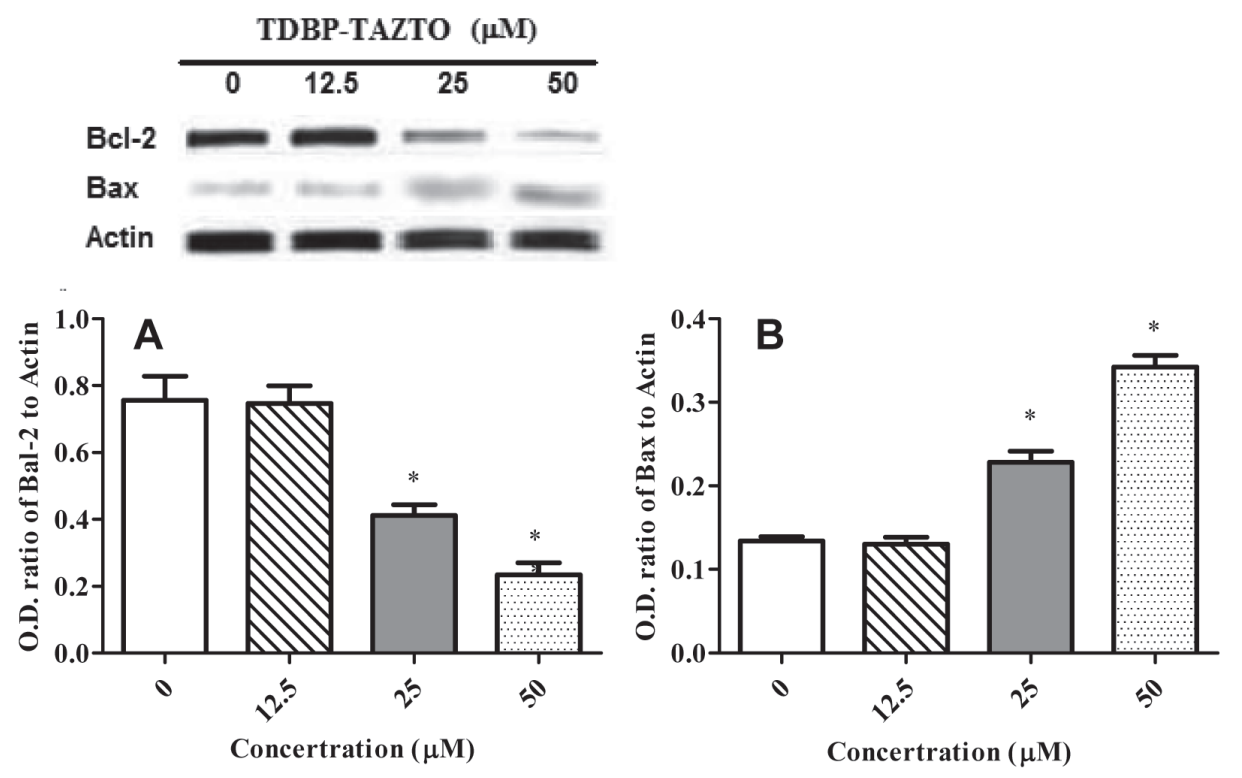

Fig. 6. The effects of TDBP-TAZTO on apoptosis-related proteins in SH-SY5Y cells (3 separated experiments). The proteins (20$30 \mu \mathrm{g}$ per lane) in cell extracts were separated by SDS polyacrylamide gel electrophoresis, and transferred onto a nitrocellulose membrane. These membranes were reacted overnight at $4^{\circ} \mathrm{C}$ with the rabbit antibody $(1: 1000)$ against $\mathrm{Bcl}-2$ and Bax. After repeated washings, the immunoreactive bands were reacted with horseradish peroxidase-conjugated anti-rabbit antibody. The O.D. value of reactive bands visible was determined densitometrically. $\beta$-actin was used as internal control. The results are presented as mean \pm S.E. ${ }^{*} p<0.05$, vs. control group. 
TDBP-TAZTO induces depression-like behaviors and neurotoxicity

Table 2. The effects of TBC on oxidative and anti-oxidative enzymes in SH-SY5Y cells.

\begin{tabular}{lccc}
\hline Group & MDA (nmol/mg pro) & GSH (nmol/mg pro) & SOD (U/mg pro) \\
\hline Control & $0.79 \pm 0.03$ & $15.05 \pm 0.13$ & $37.54 \pm 0.53$ \\
TBC $12.5 \mu \mathrm{M}$ & $1.12 \pm 0.17^{*}$ & $10.32 \pm 0.25^{*}$ & $30.01 \pm 0.63^{*}$ \\
TBC $25 \mu \mathrm{M}$ & $1.92 \pm 0.20^{*}$ & $7.62 \pm 0.18^{*}$ & $21.78 \pm 0.86^{*}$ \\
TBC $50 \mu \mathrm{M}$ & $2.42 \pm 0.21^{*}$ & $5.82 \pm 0.27^{*}$ & $16.52 \pm 0.58^{*}$ \\
\hline
\end{tabular}

Note: The results are presented as mean \pm S.E. of 3 separated experiments. ${ }^{*} p<0.05$, vs. control group.

er journal). In the present study, our further investigation found that the exposure of TDBP-TAZTO can induce significant depression-like behaviors in mice and neurotoxicity in SH-SY5Y cells, and lead to the oxidative stress and apoptosis in hippocampus and SH-SY5Y cells.

FST and TST are widely used for evaluating depression-like activity and the immobility reflects behavioral despair similar to human depression (Venna et al., 2009). In the present study, after the exposure for 30 days, TDBP-TAZTO of 5 and $50 \mathrm{mg} / \mathrm{kg}$ significantly increased immobility time with a dose-dependent manner in FST and TST, but did not affect locomotor activity, suggesting that TDBP-TAZTO exerted a significant depressionlike effect without influencing normal activity. These data suggested that TDBP-TAZTO can result in depressionlike behaviors in mice.

Depression is closely related to histopathological changes in the hippocampus in both human and rodent models (Diniz et al., 2014; Wang and Blazer, 2015). The hippocampus is vulnerable to kinds of injuries. Oxidative stress has been identified as the common and basic mechanisms causing depression (Bakunina et al., 2015; Hurley and Tizabi, 2013), and apoptosis has been implicated in depression-like disorders (Rao et al., 2012). Diverse environmental factors such as POPs and air pollution can induce oxidative stress and cell apoptosis leading to CNS pathology including depression.

Some BFRs such as pentabrominated diphenyl ether (DE-71 and PBDE-47) were found to enhance the production of reactive oxygen species (ROS). These studies showed that PBDE-47 could decrease SOD activity, GSH content and increase MDA content, which indicated PBDE-47 could cause oxidative stress. The increased free radical generation by impaired endogenous antioxidant mechanisms could induce oxidative stress, which is an important factor that has been implicated in neuronal damage (Chen et al., 2010). High oxidative stress or low antioxidant status has been implicated in the pathogenesis of depression (Palta et al., 2014; Bakunina et al., 2015). The oxidative stress can cause damage to DNA and mem- brane fatty acids and adversely affect gene expression and proteolysis, which also contribute to neurodegeneration. In order to assess whether TDBP-TAZTO could induce oxidative stress, we measured GSH content, and SOD activity, all of which are often used to reflect oxidative stress. When the antioxidants such as GSH and SOD are decreased, a large number of free radicals are generated, resulting in lipid peroxidation. As a lipid peroxidation product, MDA is also used to reflect oxidative stress injury. In our study, TDBP-TAZTO decreased GSH level and SOD activity, and increased MDA level not only in hippocampal region of mice after the exposure for 30 days, but also in the SH-SY5Y cells. These results provides the strong evidences that TDBP-TAZTO can induce oxidative stress by impairing the oxidative-antioxidant balance in neuronal cells. Oxidative stress might be one of the neurotoxic mechanisms of TDBP-TAZTO.

Apoptosis has been implicated in depression-like disorders (Rao et al., 2012). The most common pathways of proapoptosis, the intrinsic pathway is activated within the cell itself in response to oxidative stress. The intrinsic pathway from mitochondria is regulated by the $\mathrm{Bcl}-2$ family of proteins upstream of caspase activation. Bcl-2 is an apoptosis suppressing factor that heterodimerizes with pro-apoptotic protein Bax and neutralize the effect of the latter. Bcl-2 is present in excess, and cells are protected against apoptosis, while Bax is excess and the homodimers of Bax dominate, cells are susceptible to apoptosis (Yu et al., 2008). Therefore, the ratio of $\mathrm{Bcl}-2 / \mathrm{Bax}$ is a crucial factor in determining the progress of cell apoptosis. In the present study, the decreased Bcl-2 and increased Bax in the mouse hippocampus were induced by TDBP-TAZTO. Also, TDBP-TAZTO induced SH-SY5Y neuronal cell damage and apoptosis in a dose-dependent manner, which was characterized by DNA fragmentation, the increased Bax level and the decreased bcl-2 level. These results verified that TDBP-TAZTO could cause neuronal cells toxicity by inducing apoptosis via an intrinsic pathway.

The generation of ROS has been shown to be an important apoptotic signal. ROS can induce oxidative stress 
and cause damage to cell membrane and subsequently, the oxidative stimulus may trigger the intrinsic signaling pathway of cell apoptosis, which might be regulated by the anti-apoptotic protein $\mathrm{Bcl}-2$ and pro-apoptotic protein Bax in the mitochondria. We hypothesized that TDBPTAZTO can generate ROS, initiating the intrinsic apoptosis pathway, which induced neuronal cell apoptosis in the mouse hippocampus and in SH-SY5Y cells. However, one drawback of this study is that the molecular mechanisms of TDBP-TAZTO were not evaluated sufficiently. Future studies investigating the molecular mechanism underlying TBC-induced oxidative stress and apoptosis are warranted.

In summary, this study demonstrated that 30-day TDBP-TAZTO exposure induced depressive-like behaviors in mice for the first time. We found that TDBP-TAZTO resulted in upregulation of oxidative stress makers and overexpression of pro-apoptotic proteins in the mouse hippocampus. Furthermore, we also observed that TDBPTAZTO damaged SH-SY5Y cells, and led to cell apoptosis and oxidative stress. Therefore, cell apoptosis and oxidative stress are critical mechanisms in neurotoxicity of TDBP-TAZTO.

\section{ACKOWLEDGMENTS}

This work was supported by the Natural Science Foundation of Shandong Province, China (No. ZR2014HM091), the Scientific Research Foundation of Binzhou Medical College, Shandong Province, China (No. BY2013KYQD21) and the Outstanding Young Scientist Research Award Foundation of Shandong Province (No.BS2014YY008). We thank Shandong Luye Pharmaceutical Co. Ltd. for providing some experiments conditions including the laboratory and some reagents.

Conflict of interest---- The authors declare that there is no conflict of interest.

\section{REFERENCES}

Bakunina, N., Pariante, C.M. and Zunszain, P.A. (2015): Immune mechanisms linked to depression via oxidative stress and neuroprogression. Immunology, doi: 10.1111/imm.12443.

Chen, J., Liufu, C., Sun, W., Sun, X. and Chen, D. (2010): Assessment of the neurotoxic mechanisms of decabrominated diphenyl ether (PBDE-209) in primary cultured neonatal rat hippocampal neurons includes alterations in second messenger signaling and oxidative stress. Toxicol. Lett., 192, 431-439.

Dingemans, M.M., van den Berg, M. and Westerink, R.H. (2011): Neurotoxicity of brominated flame retardants: (in)direct effects of parent and hydroxylated polybrominated diphenyl ethers on the (developing) nervous system. Environ. Health. Perspect., 119, 900-907.
Diniz, B.S., Reynolds, C.F. 3rd., Begley, A., Dew, M.A., Anderson, S.J., Lotrich, F., Erickson, K.I., Lopez, O., Aizenstein, H., Sibille, E.L. and Butters, M.A. (2014): Brain-derived neurotrophic factor levels in late-life depression and comorbid mild cognitive impairment: a longitudinal study. J. Psychiatr. Res., 49, 96-101.

Feng, J., Wang, Y., Ruan, T., Qu, G. and Jiang, G. (2010): Simultaneous determination of hexabromocyclododecanes and tris (2,3dibromopropyl) isocyanurate using LC-APCI-MS/MS. Talanta, 82, 1929-1934.

Galdino, P.M., Nascimento, M.V., Sampaio, B.L., Ferreira, R.N., Paula, J.R. and Costa, E.A. (2009): Antidepressant-like effect of Lafoensia pacari A. St.-Hil. ethanolic extract and fractions in mice. J. Ethnopharmacol., 124, 581-585.

Hurley, L.L. and Tizabi, Y. (2013): Neuroinflammation, neurodegeneration, and depression. Neurotox. Res., 23, 131-144.

Kajta, M. and Wójtowicz, A.K. (2013): Impact of endocrine-disrupting chemicals on neural development and the onset of neurological disorders. Pharmacol. Rep., 65, 1632-1639.

Li, J., Liang, Y., Zhang, X., Lu, J., Zhang, J., Ruan, T., Zhou, Q. and Jiang, G. (2011): Impaired gas bladder inflation in zebrafish exposed to a novel heterocyclic brominated flame retardant tris(2,3-dibromopropyl) isocyanurate. Environ. Sci. Technol., 45, 9750-9757.

Li, J., Zhang, X., Bao, J., Liu, Y., Li, J., Li, J., Liang, Y., Zhang, J. and Zhang, A. (2015): Toxicity of new emerging pollutant tris(2,3-dibromopropyl) isocyanurate on BALB/c mice. J. Appl. Toxicol., 35, 375-382.

Nakajima, A., Saigusa, D., Tetsu, N., Yamakuni, T., Tomioka, Y. and Hishinuma, T. (2009): Neurobehavioral effects of tetrabromobisphenol A, a brominated flame retardant, in mice. Toxicol. Lett., 189, 78-83.

Palta, P., Samuel, L.J., Miller, E.R.3rd and Szanton, S.L. (2014): Depression and oxidative stress: results from a meta-analysis of observational studies. Psychosom. Med., 76, 12-19.

Qu, G.B., Shi, J.B., Li, Z.N., Ruan, T., Fu, J.J., Wang, P., Wang, T. and Jiang G.B. (2011): Detection of tris-(2, 3-dibromopropyl) isocyanurate as a neuronal toxicant in environmental samples using neuronal toxicity-directed analysis. Sci. China Chem., 54, 1651-1658.

Rao, J.S., Kellom, M., Kim, H.W., Rapoport, S.I. and Reese, E.A. (2012): Neuroinflammation and synaptic loss. Neurochem. Res., 37, 903-910.

Ruan, T., Wang, Y., Wang, C., Wang, P., Fu, J., Yin, Y., Qu, G., Wang, T. and Jiang, G. (2009): Identification and evaluation of a novel heterocyclic brominated flame retardant tris(2,3-dibromopropyl) isocyanurate in environmental matrices near a manufacturing plant in southern China. Environ. Sci. Technol., 43, 30803086.

Venna, V.R., Deplanque, D., Allet, C., Belarbi, K., Hamdane, M. and Bordet, R. (2009): PUFA induce antidepressant-like effects in parallel to structural and molecular changes in the hippocampus. Psychoneuroendocrinology, 34, 199-211.

Wang, S. and Blazer, D.G. (2015): Depression and cognition in the elderly. Annu. Rev. Clin. Psychol., 11, 331-360.

Wang, T., Han, S., Ruan, T., Wang, Y., Feng, J. and Jiang, G. (2013): Spatial distribution and inter-year variation of hexabromocyclododecane (HBCD) and tris-(2,3-dibromopropyl) isocyanurate $(\mathrm{TBC})$ in farm soils at a peri-urban region. Chemosphere, 90, 182-187.

Ye, L., Hu, Z., Du, G., Zhang, J., Dong, Q., Fu, F. and Tian, J. (2012): Antidepressant-like effects of the extract from Cimicifuga foetida L. J. Ethnopharmacol., 144, 683-691. 
TDBP-TAZTO induces depression-like behaviors and neurotoxicity

Yu, K., He, Y., Yeung, L.W., Lam, P.K., Wu, R.S. and Zhou, B. (2008): DE-71-induced apoptosis involving intracellular calcium and the Bax-mitochondria-caspase protease pathway in human neuroblastoma cells in vitro. Toxicol. Sci., 104, 341-351.

Zhang, X., Li, J., Chen, M.J., Wu, L., Zhang, C., Zhang, J., Zhou, Q.F. and Liang, Y. (2011): Toxicity of the brominated flame retardant tris-(2,3-dibromopropyl) isocyanurate in zebrafish (Danio rerio). Chinese Sci. Bull., 56, 1548-1555.

Zhu, N., Li, A., Wang, T., Wang, P., Qu, G., Ruan, T., Fu, J., Yuan, B., Zeng, L., Wang, Y. and Jiang, G. (2012): Tris(2,3-dibromopropyl) isocyanurate, hexabromocyclododecanes, and polybrominated diphenyl ethers in mollusks from Chinese Bohai Sea. Environ. Sci. Technol., 46, 7174-7181. 